\title{
Preditores do desenvolvimento social na infância: Potencial e limitações de um modelo conceitual ${ }^{1}$
}

\author{
Patrícia Alvarenga \\ Cesar A. Piccinini \\ Universidade Federal do Rio Grande do Sul
}

\begin{abstract}
RESUMO
O modelo conceitual proposto por G. R. Patterson e colegas destaca o impacto das práticas educativas parentais no desenvolvimento social infantil, e enfatiza a importância da investigação do temperamento infantil e da responsividade materna, para a compreensão das origens desse processo. No presente estudo, o potencial e as limitações desse modelo são discutidos, à luz das evidências retratadas pela literatura. A análise desse material indica o potencial do modelo conceitual, bem como a necessidade de refinamento, particularmente nos seguintes aspectos: a) estabelecimento das dimensões do temperamento e da responsividade relevantes para a compreensão do desenvolvimento social; b) determinação do impacto do uso combinado de práticas coercitivas e não-coercitivas e da contingência destas práticas; c) análise do papel do contexto da relação pais-criança em que as práticas se inserem. Para tanto se sugere a ampliação dos estudos atuais com a utilização de diversos tipos de delineamento que contribuirão para esclarecer qual a dinâmica de interação desses fatores, evitando-se a assunção simplista de relações lineares e diretas.
\end{abstract}

Palavras-chave: temperamento; responsividade; práticas educativas parentais.

\begin{abstract}
Predictors of social development in childhood: Potential and limitations of a conceptual model

The conceptual model developed by G.R. Patterson and colleagues highlights the impact of childrearing practices on children's social development and emphasizes the importance of investigating child temperament and maternal responsiveness in order to understand the origins of this process. In the present study, the potential and the limitations of this conceptual model are discussed through the analysis of some literature findings. The analysis of this material suggests both the potential of the conceptual model and its particular need for refinement of the following aspects: a) finding out the temperament and responsiveness dimensions relevant to the comprehension of social development; b) defining the effects of the combined use of coercive and noncoercive practices and of contingency on the use of these practices; c) analyzing the context of parent-child relationship in which these practices occur. Therefore, this study suggests the use of other types of research designs in order to make clear the interaction of these factors, thus avoiding simplistic assumptions about linear and direct relations.
\end{abstract}

Keywords: temperament; responsiveness; childrearing practices.

Fatores relacionados à interação pais-criança e ao contexto familiar têm sido enfatizados em estudos teóricos e empíricos sobre o desenvolvimento social nos primeiros anos da infância. Esses estudos indicam o caráter dinâmico da interação pais-criança e, em particular, da ligação entre as práticas educativas parentais e indicadores da competência social, relacionada à empatia, assertividade e à cooperação, e os indicadores de problemas de externalização, que envolvem queixas como agressividade, desobediência e comportamento desafiador. Práticas educativas pa- rentais coercitivas, e um padrão de interação familiar de caráter coercitivo têm sido sistematicamente associados aos problemas de externalização em crianças na fase pré-escolar (Alvarenga, 2004; Alvarenga \& Piccinini, no prelo; Eddy, Leve \& Fagot, 2001). Por outro lado, o uso de práticas não-coercitivas, do reforçamento positivo contingente, e o envolvimento parental positivo têm estado relacionados ao desenvolvimento da competência social (Dumas \& LaFreniere, 1993; Patterson, Reid \& Dishion, 1992). Os estudos mais recentes apontam para a necessidade de que 
sejam conduzidas investigações que permitam traçar as origens do processo de interação desses fatores (Kandel \& Wu, 1998; Keenan \& Shaw, 1998; Patterson, De Garmo \& Knutson, 2000).

Alguns estudos indicam que o nível de responsividade dos pais ao comportamento dos seus bebês seria um forte preditor do tipo e da eficácia das práticas educativas que serão empregadas no futuro. Pais menos hábeis e com menor disponibilidade emocional para perceber os sinais de seus bebês e responder adequadamente a eles tenderiam a apresentar maior dificuldade de regular o comportamento de seus filhos no futuro, empregando com maior freqüência práticas coercitivas e pouco contingentes. Esse tipo de manejo reforçaria os comportamentos inadequados da criança e exigiria dos pais práticas progressivamente mais coercitivas e severas, configurando um padrão coercitivo de interação familiar, que a literatura demonstra estar relacionado aos problemas de externalização. Por outro lado, pais mais sensíveis ao comportamento de seus bebês, tenderiam a ser mais hábeis na regulação do comportamento dos filhos em etapas posteriores do desenvolvimento, revelando maior capacidade de utilizar estratégias assertivas, contingentes e reforçadoras. Esse padrão de interação, por sua vez, favoreceria o desenvolvimento da competência social, bem como seria eficaz para reduzir a freqüência de comportamentos inadequados (Keenan \& Shaw, 1998; Patterson e cols., 2000; Patterson e cols., 1992).

Examinando as características e contribuições da criança para a interação, o temperamento infantil aparece também, tanto como um preditor da vulnerabilidade da criança para os problemas de externalização, quanto como um possível preditor da quantidade e do tipo de cuidado que os pais irão lhe prover (Boom, 1994). Crianças com características de temperamento difícil apresentariam maior tendência a desenvolver problemas de externalização (Guerin, Gottfried \& Thomas, 1997; Sanson, Hemphill \& Smart, 2004), bem como a receber cuidados menos responsivos de seus pais (Belsky, 1984). Crianças com características de temperamento fácil, apresentariam maior tendência tanto para receber cuidados mais responsivos dos pais (Belsky, 1984), como para desenvolver comportamentos socialmente adequados (Eisenberg e cols., 1993).

O modelo conceitual de Patterson e cols. (2000, 1992) permite a articulação desses cinco fatores: temperamento, responsividade, práticas educativas parentais, competência social e problemas de externalização. O modelo da coerção (Patterson e cols., 1992) ou teoria da coerção (Granic \& Patterson, 2006) explica as origens do comportamento anti-social a partir das interações que ocorrem no contexto familiar. O modelo foi desenvolvido para a compreensão do comportamento anti-social, porém, tem sido utilizado também como referencial teórico em estudos que examinam o desenvolvimento da competência social (Patterson e cols., 1992). O pressuposto central é o de que pais e crianças treinam uns aos outros a se comportar no sentido de aumentar a probabilidade de a criança desenvolver comportamentos agressivos e desafiadores, e do controle dos pais sobre esse tipo de comportamento diminuir progressivamente. Essas interações são caracterizadas por exigências de obediência dos pais seguidas pela recusa da criança em se comportar da forma exigida. Diante da inabilidade dos pais de lidar com a recusa, o comportamento da criança tende a assumir um caráter aversivo (ex. irritabilidade, agressividade, crises de birra ou oposicionismo), que tende a gradualmente agravar-se, até que o pai ou a mãe desista da exigência. Assim, através desse tipo de contingência, o comportamento da criança seria reforçado negativamente pela desistência dos pais, tornando-se mais freqüente e intenso.

Contudo o modelo não inclui apenas variáveis relacionadas à interação da díade pai/mãe-criança. De acordo com Patterson e cols. (1992) e Granic e Patterson (2006) a interação pais-criança sofre influência de inúmeros fatores contextuais e individuais. Assim, além do temperamento infantil e da responsividade parental, há outros fatores considerados preditores do comportamento anti-social. Entre eles podem ser citadas as características de personalidade dos pais (ex. comportamento anti-social e depressão materna), eventos estressores (ex. desemprego, dívidas, divórcio, etc.), ausência de suporte social adequado, e nível educacional e socioeconômico inferiores (Granic \& Patterson, 2006).

Além disso, o padrão anti-social inicialmente aprendido no contexto familiar não seria mantido ou agravado apenas pelos membros da família. O comportamento coercitivo da criança produz uma reação no ambiente social que, em geral, é manifestada através da rejeição dos pais e dos pares. Além disso, essas crianças tendem a apresentar dificuldades de aprendizagem e fracasso acadêmico (Patterson, DeBaryshe \& Ramsey, 1989). Diante dessa combinação de fatores, o indivíduo seria induzido a ligar-se a grupos de pares que também apresentam problemas de comportamento (Cairns, Cairns, Neckrtman, Gest \& Gariépy, 1988; Capaldi, Chamberlain \& Patterson, 1997; Patterson e 
cols., 1989), o que contribuiria para a chamada "escalada” do padrão anti-social.

Os estudos publicados por Patterson e colegas revelam diversas evidências do impacto das práticas educativas parentais no desenvolvimento social infantil, e referem à importância de pesquisas sobre a interação pais-criança nos primeiros meses de vida, enfatizando a avaliação do temperamento e da responsividade, para a compreensão das origens desse processo. No presente estudo, o potencial e as limitações desse modelo conceitual serão discutidos, através da apresentação de uma revisão de alguns achados da literatura sobre a dinâmica das relações entre esses fatores. Inicialmente serão abordados alguns estudos sobre 0 temperamento infantil e a responsividade materna, bem como o impacto desses fatores sobre o desenvolvimento social na infância. Na seqüência serão apresentados alguns aspectos referentes às implicações das características da interação familiar e das práticas parentais sobre o desenvolvimento dos problemas de externalização e da competência social. Por fim, esses achados são discutidos à luz do modelo teórico de Patterson e colegas, enfatizando o seu potencial e limitações na compreensão do desenvolvimento social na infância.

\section{Temperamento infantil e o desenvolvimento social}

O temperamento pode ser definido como um padrão inato e estável de reação que se refere a diferenças constitucionais na reatividade emocional, motora e atencional (Sanson, Hemphill \& Smart, 2002; Schwebel \& Plumert, 1999). A maioria dos pesquisadores da área concorda com a noção de que o temperamento tem bases biológicas ou constitucionais e se expressa logo após o nascimento. No entanto, há inúmeras evidências de que, assim como outras características do comportamento humano, o temperamento também sofre ação do ambiente, podendo ser por ele modificado (Boom, 1994; Bosa \& Piccinini, 1994; Crockenberg \& Smith, 2002; Wachs, 2002).

Thomas e Chess (1977), pioneiros no estudo do temperamento, propuseram nove dimensões para sua definição: nível de atividade física, ritmo, reação da criança a novas situações, adaptabilidade, intensidade da reação emocional, atenção-persistência, limiar de resposta, distratibilidade e humor. A partir das nove dimensões, o temperamento poderia ser classificado em três tipos: fácil, difícil ou de "aquecimento lento". No primeiro grupo estariam crianças com funcionamento biológico regular, humor positivo, pouca intensidade nas reações, tendência à aproximação e rápida adaptação a novas situações e demandas do ambiente. Ao contrário, crianças de temperamento difícil seriam aquelas com funcionamento biológico irregular, forte intensidade nas reações, humor negativo, tendência a afastar-se e a apresentar maiores dificuldades de adaptação a novos eventos. Por fim, o temperamento classificado como de "aquecimento lento" seria aquele característico de crianças com baixo nível de atividade e baixa intensidade nas reações, humor negativo e dificuldades de adaptação, mas podendo gradualmente demonstrar reações mais positivas em relação a novos eventos.

Vários outros modelos conceituais são fornecidos pela literatura para a compreensão do temperamento, entre eles o de Buss e Plomin (1984) e o de Rothbart, Ahadi, Hershey e Fisher (2001), que apresentam variações no que diz respeito às dimensões empregadas. Em uma revisão da literatura sobre o temperamento e suas relações com o desenvolvimento social, Sanson e cols. (2004) afirmam que três grandes dimensões do temperamento têm recebido crescente aceitação: reatividade ou emocionalidade negativa, que se refere à irritabilidade, humor negativo, e reações negativas de alta intensidade; auto-regulação, que tem dois componentes: controle da atenção e controle das emoções; e aproximação-retraimento que descreve a tendência à aproximação ou ao retraimento diante de novas situações e pessoas. Uma quarta dimensão envolveria o ritmo e o nível de atividade física, entretanto, segundo esses autores, ela não tem se mostrado significativa. No entanto, vários estudos indicam relações importantes entre o alto nível de atividade física e os problemas de externalização (Colder, Mott \& Berman, 2002; Gjone \& Stevenson, 1997; Hagekull, 1994).

No que diz respeito às relações entre o temperamento infantil e indicadores de competência social, as dimensões emocionalidade negativa e auto-regulação têm se destacado. Por exemplo, Eisenberg e cols. (1993) mostraram que a capacidade de auto-regulação e a emocionalidade negativa estavam fortemente relacionadas a uma série de habilidades sociais relatadas pelos pais e professores e também observadas nas crianças. A alta emocionalidade negativa foi um fator de risco para o déficit em habilidades sociais tanto para meninos como para meninas, enquanto a baixa emocionalidade negativa foi um fator protetivo, porém, apenas para os meninos. A presença de interações entre as dimensões avaliadas foi sugerida, pois foi observado que tanto os meninos quanto as meninas que apresentavam pouca capacidade de auto-regulação e alta emocionalidade negativa tiveram os maiores 
déficits em habilidades sociais. Outro estudo interessante foi conduzido por Lehman, Steier, Guidash e Wanna (2002), que investigou a influência de características de temperamento de crianças de 15 a 31 meses de idade sobre a obediência a solicitações de suas mães durante a tarefa de guardar brinquedos. As crianças percebidas pelas mães como menos receosas em relação a situações sociais novas e com menor predisposição à raiva foram mais cooperativas durante a tarefa.

Nas pesquisas sobre as relações entre temperamento infantil e problemas de externalização, encontram-se muitas evidências sobre o papel da emocionalidade negativa e do nível de atividade física. Por exemplo, o estudo de Gjone e Stevenson (1997) verificou que elevados níveis de emocionalidade negativa foram preditores de comportamento delinqüente e comportamento agressivo em crianças. Além disso, altos escores na dimensão atividade física também foram preditores de comportamento agressivo. Nessa mesma perspectiva, o estudo de Colder e cols. (2002) revelou que alto nível de atividade e baixo nível de medo no primeiro ano de vida foram preditores de problemas de externalização dos 4 aos 8 anos. Contudo, o temperamento não foi preditor de problemas de externalização nas meninas. No estudo de Hagekull (1994), a emocionalidade negativa, juntamente com a impulsividade e o nível de atividade física foram preditores dos problemas de externalização aos 4 anos. No estudo de Shaw, Owens, Giovanelli e Winslow (2001) crianças que apresentavam sintomas do transtorno da conduta ou do transtorno opositivo desafiador aos 6 anos de idade haviam apresentado um padrão de emocionalidade negativa entre 18 e 24 meses. Outras relações foram encontradas por Guerin e cols. (1997), que verificaram que a dificuldade de adaptação, a resistência ao controle e a dificuldade de socialização estiveram positivamente correlacionadas com problemas de externalização dos 4 aos 12 anos. A dimensão resistência ao controle, verificada nos primeiros anos, também apresentou uma correlação moderada com os problemas de externalização verificados dos 5 aos 10 anos no estudo de Bates, Pettit, Dodge e Ridge (1998).

Outros estudos referem relações entre o temperamento classificado como difícil (de acordo com o modelo de Thomas e Chess, 1977) e os problemas de externalização. Maziade e cols. (1990) utilizaram um delineamento longitudinal para investigar essa hipótese e verificaram que o temperamento difícil foi preditor de altos escores em problemas de externalização em crianças de 3 a 12 anos. Os achados de Guerin e cols. (1997) apóiam a mesma hipótese. O estudo examinou o ajustamento comportamental em pré-escolares, revelando que crianças descritas pelas mães como mais difíceis de serem cuidadas aos 18 meses apresentaram mais problemas de externalização dos 3 aos 12 anos.

\section{Responsividade materna e o desenvolvimento social}

O conceito de sensibilidade materna tem sido bastante usado na literatura para se referir à atenção e percepção consistentes, interpretação acurada e resposta contingente e apropriada da mãe aos sinais da criança (Isabella, Belsky \& von Eye e cols. 1989; Boom, 1994). Essa característica da mãe favoreceria a ocorrência de interações sincrônicas e mutuamente recompensadoras entre a díade. Já Wakschlag e Hans (1999), destacaram o conceito de responsividade que seria distinto de outros atributos parentais positivos, como o afeto e a estimulação, porque envolveria ações parentais que são contingentes a sinais da criança e que são apropriadas em relação aos seus desejos e necessidades, incluindo sua necessidade de autonomia. De acordo com esses autores, a responsividade não seria um construto único, mas um domínio que consiste em um complexo de variáveis relacionadas, como, por exemplo, sensibilidade a pistas sociais, empatia, capacidade de previsão, não-intrusividade, disponibilidade emocional e envolvimento positivo.

Mães pouco responsivas seriam, portanto, menos contingentes ao reagirem aos comportamentos de seus bebês, bem como menos adequadas ao responderem. Essas mães teriam menor capacidade de facilitar a regulação emocional da criança (Goodman \& Gotlib, 1999), bem como maior tendência de deixar de reforçar contingentemente respostas positivas do bebê, como a expressão de afeto e tentativas de comunicação. Nestes casos, é comum observar as mães responderem com maior freqüência e prontidão, a comportamentos dos bebês que indicam estresse e desconforto, do que a comportamentos positivos. Na perspectiva de Patterson e cols. (1992), seria possível interpretar esta etapa como um estágio inicial do desenvolvimento de padrões disfuncionais de interação, caracterizado pelo reforçamento e conseqüente intensificação de comportamentos aversivos na criança, com prejuízos para o desenvolvimento de comportamentos positivos. A criança desde os primeiros meses de vida estaria aprendendo ativamente que através de comportamentos aversivos como o choro e outras 
formas de protesto e sinalização de estresse, ela obtém mais atenção e cuidados. O estudo de Alvarenga \& Piccinini (no prelo) apoiou parcialmente estas relações ao mostrar que a responsividade foi preditora da sensibilidade materna, definida como comportamentos que indicam atenção, consideração e respeito em relação às necessidades, desejos ou vontades da criança.

A responsividade também tem sido considerada um importante preditor do desenvolvimento social da criança. Wakschlag e Hans (1999) encontraram relações positivas significativas entre baixa responsividade materna entre 4 e 24 meses, e o transtorno opositivo desafiador e o transtorno de conduta em uma amostra de risco de crianças de 10 anos. No entanto, no estudo de Keenan e Shaw (1998) os resultados revelaram que, somente para os meninos, o nível de responsividade materna aos 12 meses, juntamente com o nível de agressividade global da criança aos 18 meses, foram preditores significativos de agressividade aos 24 meses. Para as meninas essa associação não ocorreu.

De modo similar, alguns estudos que investigaram antecedentes da competência social na interação mãecriança apontam a responsividade como um importante preditor desta característica. O estudo de Landry, Smith, Swank, Assel e Vellet (2001) apresentou evidências da importância da responsividade materna para o desenvolvimento da competência social na infância em crianças prematuras e crianças nascidas a termo. Tanto a responsividade das mães, quanto as habilidades sociais das crianças foram avaliadas quando as crianças tinham 6, 12 e 24 meses, e novamente quando completaram 3 e 4 anos de vida. Diferentes indicadores das habilidades sociais foram utilizados em cada uma das etapas da coleta de dados, incluindo o número de vocalizações, olhares e gestos nas primeiras etapas, e medidas de obediência e negociação nas etapas finais. Crianças pré-termo com mães pouco responsivas apresentaram níveis de habilidades sociais significativamente mais baixos do que crianças pré-termo com mães responsivas. Para as crianças nascidas a termo, não houve diferenças no nível de habilidades sociais entre os grupos de alta e baixa responsividade materna. Porém, foi constatado que o desenvolvimento das habilidades sociais das crianças do grupo de baixa responsividade foi mais lento. Além disso, todas as crianças apresentaram maior desaceleração no desenvolvimento social, quando suas mães eram pouco responsivas.

Belsky e Pasco Fearon (2002) investigaram o tipo de apego em bebês aos 15 meses (que é indicador do nível de responsividade materna) e a responsividade materna aos 24 meses da criança como possíveis preditores de problemas de comportamento e competência social no $3^{\circ}$ ano de vida. Os resultados revelaram que crianças com histórico de apego seguro aos 15 meses (indicador de alta responsividade) e alta responsividade materna também aos 24 meses apresentaram os melhores índices de competência social e os menores escores de problemas de comportamento. $\mathrm{O}$ grupo de crianças com histórico inconsistente (ex. baixa responsividade aos 15 meses e alta responsividade aos 24 meses), apresentou desempenho inferior ao do grupo com histórico consistente de alta responsividade, mas superior ao das crianças com histórico de baixa responsividade materna tanto aos 15 , quanto aos 24 meses. Analisando também o histórico de responsividade, o estudo de Landry e cols. (2001) mostrou que o desenvolvimento social foi similar entre as crianças que tinham mães consistentemente responsivas (alta responsividade nos primeiros meses de vida e alta responsividade no $3^{\circ}$ e $4^{\circ}$ ano de vida) e as crianças que tinham mães que apresentavam responsividade inconsistente. Porém, constatou-se uma desaceleração no desenvolvimento social das crianças do grupo de responsividade inconsistente no quarto ano de vida.

\section{Práticas educativas e desenvolvimento social}

De acordo com Patterson e cols. (1992), o conceito chave para a compreensão do desenvolvimento social no contexto familiar é o de contingência das práticas educativas parentais. Esse conceito faz referência ao fato de os pais comportarem-se efetiva e consistentemente no sentido de reduzir comportamentos inadequados e coercitivos, e de estimular a ocorrência de comportamentos socialmente adequados. $\mathrm{O}$ resultado de práticas educativas contingentes seria o de fornecer à criança, entre outras coisas, previsibilidade e, portanto, organização ao seu repertório comportamental. Em um ambiente contingente, a criança, na maior parte do tempo, sabe que comportamentos levarão a conflito e que comportamentos produzem encorajamento e afeto. O uso de práticas contingentes poderia conduzir ao desenvolvimento da competência social de duas formas distintas. Práticas que, além de contingentes, incluem o uso do reforço positivo para comportamentos socialmente desejáveis, envolvimento positivo dos pais com os filhos e ordens e pedidos claros e diretos à criança, estariam associadas ao desenvolvimento da competência social e da auto-estima. Entretanto, crianças submetidas a práticas contingentes, mas que priorizam o uso do desprezo, da culpa e 
da ansiedade para atingir os objetivos de socialização, poderiam também desenvolver competência social, porém, com baixa auto-estima e possíveis problemas de internalização, entre eles, ansiedade e depressão.

Além das práticas contingentes, o envolvimento parental é outro conceito utilizado por Patterson e cols. (1992) para a compreensão da competência social. Esse conceito refere-se ao tempo que os pais passam junto com a criança em atividades que envolvam trocas positivas entre eles. Esse seria um aspecto não contingente da relação pais-criança, que contribuiria significativamente também para o desenvolvimento da auto-estima.

Por outro lado, práticas parentais não contingentes levariam ao desenvolvimento de um repertório pobre em competência social e aos problemas de externalização. Para Patterson e cols. (1992) os membros da família treinam diretamente esses comportamentos na criança, configurando o que os autores chamam de ciclo familiar coercitivo. Os pais, em geral, não são contingentes no uso de reforçadores positivos para iniciativas pró-sociais (Dumas \& Wahler, 1985), fracassam no uso efetivo de técnicas disciplinares para enfraquecer os comportamentos indesejáveis (DeBaryshe, Patterson \& Capaldi, 1993), além de muitas vezes utilizar uma disciplina severa, embora inconsistente (Fox, Platz \& Bentley, 1995; Pettit, Bates \& Dodge, 1997).

A conseqüência de práticas parentais ineficazes é estimular interações diárias, nas quais os membros da família inadvertidamente reforçam positiva ou negativamente o comportamento coercitivo, através de atenção e aprovação, ou da interrupção de exigências e solicitações. Este tipo de funcionamento familiar faria com que, na medida em que os comportamentos da criança se agravam, as estratégias de controle dos pais se tornassem progressivamente mais coercitivas e severas, embora ineficazes (Capaldi e cols., 1997).

A maioria dos estudos que investiga as relações entre a competência social e as práticas educativas parentais confirma a importância do uso de práticas não-coercitivas no geral (ex. explicações, negociações), do reforçamento positivo contingente e do envolvimento positivo dos pais com a criança (Arsenio, 2004; Raver, 1996). Por exemplo, Crockenberg e Littman (1990) investigaram a relação entre as práticas educativas maternas e a assertividade de crianças com 2 anos de idade. Foram encontradas correlações positivas da assertividade com o uso de comandos diretos e do reforçamento contingente, e com o uso de orientações e sugestões. No entanto, foi encontrada também uma correlação positiva entre a assertividade e o controle negativo. No estudo de Dumas e LaFreniere (1993), constatou-se que mães de crianças consideradas socialmente competentes foram mais positivas em suas interações com os filhos, através de demonstrações de aprovação e palavras e gestos de carinho, e menos coercitivas, quando comparadas às mães de crianças consideradas ansiosas ou agressivas, durante a interação em uma tarefa estruturada. Além disso, as mães do grupo de crianças socialmente competentes utilizaram menos comandos, sendo menos intrusivas, quando comparadas aos outros dois grupos.

Nesta mesma perspectiva, o estudo de Denham, Renwick e Holt (1991) investigou alguns preditores maternos da assertividade e de outros comportamentos sociais positivos como a empatia, o altruísmo e a cooperação em pré-escolares. Os achados indicaram que as emoções positivas e a autorização da autonomia pelas mães estiveram positivamente correlacionadas à assertividade da criança. O estudo de Raver (1996) investigou a influência da reciprocidade e da atenção compartilhada na interação mãe-criança, sobre a empatia (diante de um suposto ferimento do experimentador) e a auto-regulação emocional (durante atraso no recebimento de uma recompensa), que foram considerados indicadores de competência social de crianças aos 2 anos de idade. Os resultados mostraram que os dois fatores preditores correlacionaram-se de forma positiva e significativa apenas à auto-regulação emocional.

O impacto das práticas educativas parentais sobre a obediência durante a infância não é tão claro, pois a literatura apresenta achados controversos a esse respeito. Alguns estudos mostram que estratégias de controle fraco a moderado estão tipicamente associadas à obediência. No entanto, outras investigações sugerem que práticas de caráter coercitivo ou severo também estariam associadas a esse tipo de comportamento. No estudo de Crockenberg e Littman (1990) foi encontrada uma forte correlação positiva entre a obediência e o uso de comandos diretos (por exemplo, ordens e pedidos) e de reforçamento contingente. Por outro lado, a obediência também esteve positivamente correlacionada ao uso de sugestões ou orientações e do controle negativo, definido como críticas, punições físicas e ameaças. Para Kochanska (2002), a obediência pode se manifestar na infância de diferentes formas. A criança pode revelar afeto positivo e comprometimento ao obedecer (obediência comprometida), ou pode demonstrar hostilidade ou afeto neutro (obe- 
diência situacional). É possível que estas diferentes manifestações da obediência estejam relacionadas a diferentes estratégias reguladoras por parte dos pais. Enquanto a obediência comprometida estaria relacionada a práticas de controle mais fraco ou moderado (ex. sugestões e orientações), a obediência situacional estaria relacionada a estratégias de controle coercitivo, porém, contingente.

Com relação aos problemas de externalização, a maioria das investigações tem enfatizado a relação entre o uso predominante de práticas de caráter coercitivo e a falta de afeto e reforçamento positivo e contingente, e esse padrão comportamental. No estudo de McLoyd e Smith (2002), o uso de punição física esteve associado a um aumento dos problemas de comportamento em crianças que foram acompanhadas dos 4 aos 10 anos de idade, sendo que o afeto e o suporte emocional fornecidos pela mãe foram moderadores nessa relação. Fox e cols. (1995) obtiveram resultados semelhantes num estudo com crianças pré-escolares. Mais precisamente revelaram uma correlação positiva significativa entre o uso de punições físicas e a presença de problemas de comportamento, assim como uma correlação negativa entre práticas educativas não coercitivas e problemas de comportamento. Outros dois estudos, realizados com crianças brasileiras, apóiam esses achados. Ferreira e Marturano (2002) verificaram que famílias de crianças com problemas de comportamento apresentaram mais práticas punitivas e modelos adultos agressivos. No estudo de Alvarenga e Piccinini (2001) os problemas de externalização de crianças de 5 e 6 anos de idade estiveram relacionados às práticas coercitivas em geral, e também à punição física em particular.

Por outro lado, o estudo de Crockenberg e Littman (1990) revelou ligações entre práticas não-coercitivas como comandos diretos ou de reforçamento contingente, e o comportamento desafiador em crianças de 2 anos de idade. Existem outras evidências de que o uso de estratégias de controle moderadas como os comandos diretos, por exemplo, poderiam estar associadas também ao comportamento desafiador (Belsky, Woodworth \& Crnic, 1996).

\section{Articulando as evidências: potencial e limitações do modelo conceitual}

As evidências indicadas pelos estudos discutidos revelam de forma incontestável a pertinência do modelo conceitual de Patterson e cols. (1992) para a compreensão do desenvolvimento social nos primeiros anos da infância. Contudo, os achados controversos que permeiam as relações dos problemas de externalização e da competência social com cada um dos fatores preditores indicam a necessidade de aprimoramento.

Quanto ao impacto das características do temperamento infantil, a emocionalidade negativa e a atividade física em altos níveis, bem como o temperamento classificado como difícil, aparecem de forma consistente como importantes preditores dos problemas de externalização, enquanto a capacidade de auto-regulação parece constituir um fator protetivo contra o surgimento desses problemas, favorecendo a competência social. Por outro lado, existem ainda algumas dimensões, como o retraimento ou adaptação lenta diante de novas situações e o medo ou receio (social fearfulness), que aparecem em achados controversos, que ora os indicam como fatores protetivos para externalização, ora como preditores. Esses achados provavelmente refletem a diversidade tanto no que se refere à definição, como à mensuração das dimensões que compõem o construto temperamento. Embora os autores mencionados acima compartilhem algumas concepções a respeito da definição do temperamento, existem ainda muitas divergências com relação as suas dimensões. Apesar de todos os estudos realizados nas últimas duas décadas, estas questões continuam a desafiar muitos pesquisadores.

Além disso, os estudos não fornecem uma teorização consistente que explique a relação entre essas dimensões específicas e o desenvolvimento social. Por exemplo, algumas investigações consideram a hipótese de que emocionalidade negativa representaria uma predisposição para a raiva e para o comportamento agressivo (Cairns \& Cairns, 1991; Ledingham, 1991). Contudo, acerca do nível de atividade física, do retraimento diante de novas situações e do medo, essas relações, em termos conceituais, ainda não estão claras.

A responsividade materna também aparece consistentemente relacionada ao desenvolvimento social. Os estudos revisados revelam os déficits causados pela baixa responsividade no repertório social da criança, bem como indicam que a alta responsividade tende a favorecer a competência social e prevenir problemas de comportamento de um modo geral. De qualquer modo, seria interessante refletir sobre os possíveis ganhos provenientes do estudo de dimensões específicas da responsividade, que constitui, como já enfatizado acima, não um construto único, mas sim, um domínio de variáveis relacionadas, como, por exemplo, a sensibilidade a pistas sociais, a empatia e a nãointrusividade (Wakschlag \& Hans, 1999). Uma abor- 
dagem metodológica que privilegiasse a avaliação de algumas dessas dimensões, ao invés do nível de responsividade geral, seria promissora.

De modo semelhante, os achados sobre as relações entre as práticas educativas parentais e indicadores do desenvolvimento social também revelam, mais do que inconsistências, a complexidade desta problemática. Algumas das questões importantes que surgem da análise desses estudos referem-se: a) à importância do estudo de estratégias combinadas, como, por exemplo, o uso moderado de práticas coercitivas junto a práticas assertivas e ao reforçamento positivo; b) à necessidade de avaliar se as práticas são utilizadas pelos pais de forma contingente ou não; c) à relevância da análise do contexto da relação pais-criança em que determinada prática se insere. Para Crockenberg e Litman (1990) o comportamento desafiador da criança fica relacionado ao uso de comandos ou outras estratégias maternas assertivas de controle, quando estas ocorrem após uma recusa assertiva da criança (ex. dizer não a uma solicitação materna). Por exemplo, a mãe insiste em um comando direto, mesmo após a criança ter manifestado assertivamente a sua recusa em comportar-se da forma solicitada. Nesse contexto, a criança estaria menos propensa a obedecer ou cooperar e mais propensa a demonstrar comportamento desafiador, porque a mãe estaria sinalizando sua não disponibilidade para negociar, explicar a situação ou compreender o ponto de vista da criança. Assim, é provável que, nesse tipo de situação, ela opte por repetir a recusa, ignorar a solicitação da mãe ou desafiá-la.

A análise desses estudos indica o potencial do modelo de Patterson e cols. $(2000,1992)$ para a compreensão do desenvolvimento da competência social e dos problemas de externalização nos primeiros anos da infância. Contudo, embora a literatura indique inúmeras relações entre os cinco fatores aqui destacados, o modelo necessita de novas pesquisas que permitam seu refinamento, no sentido de esclarecer de que forma ocorrem essas relações, considerando-as em toda a sua complexidade, e evitando o reducionismo de posições que enfatizam relações lineares e diretas. Além disso, é necessário considerar questões conceituais e metodológicas envolvidas na avaliação e interpretação dos resultados referentes a cada um dos construtos que fazem parte do modelo. Por um lado, a descoberta das dimensões mais relevantes na investigação do temperamento, da responsividade e das práticas educativas parentais para a compreensão do desenvolvimento social, poderia resultar em avanços importantes. Por outro lado, a utilização de outros tipos de delineamento, como o estudo de caso, e o delineamento experimental de caso único, também poderia ser útil para esclarecer melhor a dinâmica de interação entre os cinco fatores que compõem o modelo conceitual aqui apresentado.

\section{REFERÊNCIAS}

Alvarenga, P. (2004). Problemas de externalização e competência social na infância: $O$ impacto do temperamento infantil, da responsividade e das práticas educativas maternas. Tese de Doutorado não-publicada, Programa de Pós-Graduação em Psicologia do Desenvolvimento, Universidade Federal do Rio Grande do Sul. Porto Alegre.

Alvarenga, P., \& Piccinini, C. A. (2001). Práticas educativas maternas e problemas de comportamento em pré-escolares. Psicologia: Reflexão e Crítica, 14(3), 449-459.

Alvarenga, P., \& Piccinini, C. A. (no prelo). O impacto do temperamento, da responsividade e das práticas educativas maternas no desenvolvimento dos problemas de externalização e da competência social. Psicologia: Reflexão e Crítica.

Arsenio, W. F. (2004). The stability of young children's physical aggression: Relations with child care, gender, and aggression subtypes. Monographs of the Society for Research in Child Development, 69(4), 130-143.

Bates, J. E., Pettit, G. S., Dodge, K. A., \& Ridge, B. (1998). Interaction of temperamental resistance to control and restrictive parenting in the development of externalizing behavior. Developmental Psychology, 34(5), 982-995.

Belsky, J. (1984). The determinants of parenting: A process model. Child Development, 55, 83-96.

Belsky, J., \& Pasco Fearon, R. M. (2002). Early attachment security, subsequent maternal sensitivity, and later child development: Does continuity in development depend upon continuity of caregiving? Attachment \& Human Development, 4(3), 361387.

Belsky, J., Woodworth, S., \& Crnic, K. (1996). Trouble in the second year: Three questions about family interaction. Child Development, 67, 556-578.

Boom, D. C. van den (1994). The influence of temperament and mothering on attachment and exploration: An experimental manipulation of sensitive responsiveness among lower-class mothers with irritable infants. Child Development, 65, 14491469.

Bosa, C. A., \& Piccinini, C. A. (1994). Temperamento infantil e apego mãe-criança: Considerações teóricas. Psicologia: Teoria e Pesquisa, 10, 193-212.

Buss, A. H., \& Plomin, R. (1984). Temperament: Early developing personality traits. Hillsdale, NJ: Erlbaum.

Cairns, R. B., \& Cairns, D. D. (1991). Social cognition and social networks: A developmental perspective. Em D. J. Pepler \& K. H. Rubin (Orgs.), The development and treatment of childhood aggression (p. 249-278). Hillsdale, NJ: Erlbaum.

Cairns, R., Cairns, B., Neckrtman, H., Gest, S., \& Gariépy, J-L. (1988). Social networks and aggressive behavior: Peer support or peer rejection? Developmental Psychology, 24, 813-823.

Capaldi, D., Chamberlain, P., \& Patterson, G. (1997). Ineffective discipline and conduct problems in males: Association, late 
adolescent outcomes and prevention. Agression and Violent Behavior, 2(4), 343-353.

Colder, C. R., Mott, J. A., \& Berman, A. S. (2002). The interactive effects of infant activity level and fear on growth trajectories of early child behavior problems. Development and Psychopathology, 14, 1-23.

Crockenberg, S. B., \& Litman, C. (1990). Autonomy as competence in 2-year-olds: Maternal correlates of child defiance, compliance and self-assertion. Developmental Psychology, 26(6), 961-971.

Crockenberg, S. B., \& Smith, P. (2002). Antecedents of motherinfant interaction and infant irritability in the first 3 months of life. Infant Behavior and Development, 25, 2-15.

DeBaryshe, B., Patterson, G., \& Capaldi, G. (1993) A performance model for academic achievement in early adolescent boys. Developmental Psychology, 29(5), 795-804.

Denham, S. A., Renwick, S. M., \& Holt, R. W. (1991). Working and playing together: Prediction of preschool social-emotional competence from mother-child interaction. Child Development, 62, 242-249.

Dumas, J., \& Wahler, R. G. (1985). Indiscriminate mothering as a contextual factor in agressive-oppositional child behavior: “Damned if you do damned if you don't”. Journal of Abnormal Child Psychology, 13, 1-18.

Dumas, J. E., \& LaFreniere, P. J. (1993). Mother-child relationships as sources of support or stress: A comparision of competent, average, agressive, and anxious dyads. Child Development, 64, 1732-1754.

Eddy, J. M., Leve, L. D., \& Fagot, B. I. (2001). Coercive family processes: A replication and extension of Patterson's coercion model. Agressive Behavior, 27, 14-25.

Eisenberg, N., Fabes, R. A., Bernzweig, J., Karbon, M., Poulon, R., \& Hanish, L. (1993). The relations of emotionality and regulation to preschoolers' social skills and sociometric status. Child Development, 64, 1418-1438.

Ferreira, M. C. T., \& Marturano, E. M. (2002). Ambiente familiar e os problemas de comportamento apresentados por crianças com baixo desempenho escolar. Psicologia: Reflexão e Crítica, 15(1), 35-44.

Fox, R. A., Platz, D. L., \& Bentley, K. S. (1995). Maternal factors related to parenting practices, developmental expectations, and perceptions of child behavior problems. The Journal of Genetic Psychology, 156, 431-441.

Gjone, H., \& Stevenson, J. (1997). A longitudinal twin study of temperament and behavior problems: Common genetic or environmental influences? Journal of the American Academy of Child and Adolescent Psychiatry, 36(10), 1448-1456.

Goodman, S. H., \& Gotlib, I. H. (1999). Risk for psychopathology in the children of depressed mothers: A developmental model for understanding mechanisms of transmission. Psychological Review, 106(3) 458-490.

Granic, I., \& Patterson, G. R. (2006). Toward a comprehensive model of antisocial development: A dynamic systems approach. Psychological Review, 113(1), 101-131.

Guerin, D. W., Gottfried, A., \& Thomas, C. W. (1997). Difficult temperament and behaviour problems: A longitudinal study from 1.5 to 12 years. International Journal of Behaviour Development, 21(1), 71-90.
Hagekull, B. (1994). Infant temperament and early childhood functioning: Possible relations to the five-factor model. Em C. J. Halverson, Jr., G. A. Kohnstamm \& R. P. Martin (Orgs.), The developing structure of temperament and personality (p. 227-240). Hillsdale, NJ: Erlbaum.

Isabella, R. A., Belsky, J., \& Von Eye, A. (1989). Origins of infant-mother attachment: An examination of interactional synchrony during the infant's first year. Developmental Psychology, 25(1), 12-21.

Kandel, D. B., \& Wu, P. (1998). Disentangling mother-child effects in the development of anti-social behavior. Em J. McCord (Org.), Coercion and punishment in long-term perspectives (pp. 106-123) Cambridge: Cambridge University Press.

Keenan, K., \& Shaw, D. (1998). The development of coercive family processes: The interaction between aversive toddler behavior and parenting factors. Em J. McCord (Org.), Coercion and punishment in long-term perspectives (p. 165-180). Cambridge: Cambridge University Press.

Kochanska, G. (2002). Commited compliance, moral self, and internalization: A mediational model. Developmental Psychology, 38(3), 339-351.

Landry, S. H., Smith, K. E., Swank, P. R., Assel, M. A., \& Vellet, S. (2001). Does early responsive parenting have a special importance for children's development or is consistency across early childhood necessary? Development Psychology, 37(3), 387-403.

Ledingham, J. E. (1991). Social cognition and aggression. Em D. J. Pepler \& K. H. Rubin (Orgs.), The development and treatment of childhood aggression (pp. 279-286). Hillsdale, NJ: Erlbaum.

Lehman, E. B., Steier, A. J., Guidash, K. M., \& Wanna, S. Y. (2002). Predictors of compliance in toddlers: Child temperament, maternal personality, and emotional availability. Early Child Development and Care, 172(3), 301-310.

Maziade, M., Caron, C., Cote, R., Merette, C., Bernier, H., Laplante, B., Boutin, P., \& Thivierge, J. (1990). Psychiatric status of adolescents who had extreme temperaments at age seven. American Journal of Psychiatry, 147, 1531-1536.

McLoyd, V. C., \& Smith, J. (2002). Phisical discipline and behavior problems in african american, european, and spanic children: Emotional support as a moderator. Journal of Marriage and Family, 64, 40-53.

Patterson, G., DeBaryshe, D., \& Ramsey, E. (1989). A developmental perspective on antisocial behavior. American Psychologist, 44, 329-335.

Patterson, G. R., DeGarmo, D. S., \& Knutson, N. (2000). Hyperactive and antisocial behaviors: Comorbid or two points in the same process? Development and Psychopathology, 12, 91-106.

Patterson, G. R., Reid, J., \& Dishion, T. (1992). Antisocial boys. Eugene: Castalia Publishing Company.

Pettit, G. S., Bates, J. E., \& Dodge, K. A. (1997). Supportive parenting, ecological context, and children's adjustment: A seven-year longitudinal study. Child Development, 68, 908923.

Raver, C. C. (1996). Relations between social contingency in mother-child interaction and 2-year-olds' social competence. Developmental Psychology, 32(5), 850-859. 
Rothbart, M. K., Ahadi, S. A., Hershey, K. L., \& Fisher, P. (2001). Investigation of temperament at three to seven years: The Children's Behavior Questionnaire. Child Development, 72(5), 1394-1408.

Sanson, A., Hemphill, S., \& Smart, D. (2002). Temperament and social development. Em P. K. Smith \& C. H. Hart (Orgs.), Handbook of childhood social development (p. 97-116). London, UK: Blackwell.

Sanson, A., Hemphill, S., \& Smart, D. (2004). Conecctions between temperament and social development: A review. Social Development, 13, 142-168.

Schwebel, D. C., \& Plumert, J. M. (1999). Longitudinal and concurrent relations among temperament, ability estimation and injury proneness. Child Development, 70, 700-712.

Shaw, D. S., Owens, E. B., Giovanelli, J., \& Winslow, E. B. (2001). Infant and toddler pathways leading to early externalizing disorders. Journal of the American Academy of Child and Adolescent Psychiatry, 40, 36-43.
Thomas, A., \& Chess, S. (1977). Temperament and development. New York: Pleno.

Wachs, T. D. (2002). "Back to the future": A commentary on Crockenberg and Smith (1982). Infant Behavior and Development, 25, 21-24.

Wakschlag, L. S., \& Hans, S. L. (1999). Relation of maternal responsiveness during infancy to the development of behavior problems in high-risk youths. Developmental Psychology, 35(2), 569-579.

\section{Nota:}

1 Este estudo foi baseado na tese de doutorado de Patrícia Alvarenga, realizada sob a supervisão de Cesar Piccinini, apresentada no Programa de Pós-Graduação em Psicologia do Desenvolvimento da UFRGS, Porto Alegre - RS.

\section{Sobre os autores:}

Patrícia Alvarenga: Doutora em Psicologia pela UFRGS e professora do Programa de Pós-Graduação em Psicologia da UFBA.

Cesar A. Piccinini: Doutor pela Universidade de Londres e professor do Programa de Pós-Graduação em Psicologia da UFRGS.

Endereço para correspondência: Patrícia Alvarenga - Departamento de Psicologia, Faculdade de Filosofia e Ciências Humanas, Universidade Federal da Bahia - Rua Aristides Novis, 197 - Federação - Salvador - BA - CEP 40210-730 - Endereço eletrônico: palvarenga66@gmail.com 